\title{
The Superior Peripheral Iridectomy: Prevention of Pupil Block due to Silicone Oil
}

\author{
A.- J. ELLIOTT, A. S. BACON, J. D. SCOTT \\ Cambridge
}

\begin{abstract}
Summary
Superior peripheral iridectomy has been performed in 40 patients who have undergone silicone oil surgery for retinal detachment and who are aphakic. Only two developed raised intraocular pressure due to pupil block by liquid silicone and in each case the iridectomies seemed to be closed by proliferative membrane rather than the silicone meniscus.

This type of iridectomy is particularly suitable in patients requiring intracapsular cataract extraction after previous vitrectomy and silicone oil surgery.
\end{abstract}

Silicone oil injection is a valuable technique in treating difficult retinal detachments because of its ability to demonstrate and separate preretinal membranes and to provide a permanent tamponade for retinal breaks. ${ }^{1-3}$ However, under certain circumstances it may also tamponade the pupil causing pupil block; continued production of aqueous then pushes silicone through the pupil against the surface tensile forces which act to keep the silicone bubble behind it. Drainage of anterior chamber aqueous continues until the chamber is filled with silicone when the intraocular pressure rises. Silicone oil mediated pupil block causing intra-ocular hypertension was first described by Ando who with other authors described the 6 o'clock position peripheral iridectomy in aphakic eyes to prevent this complication. ${ }^{4-6}$ In addition, continued presence of silicone oil in the anterior chamber having entered by this mechanism may lead to characteristic keratopathy. ${ }^{7-9}$

Lens opacities frequently develop in eyes which undergo vitrectomy and silicone oil injection. Incidences of $49 \%$ to $100 \%$ have been reported ${ }^{9,10}$ and cataract extraction is often necessary. When this is carried out through a standard cataract incision it is simpler to do superior iridectomies rather than an inferior iridectomy which requires the use of a suction cutter. However, it is believed that a superior iridectomy would be ineffective. ${ }^{5}$ This belief is based on the idea that, as silicone is less dense than aqueous, it would tend to move upwards to occlude a superiorly placed iridectomy. We report a series of patients treated with superior peripheral iridectomy.

\section{Patients and methods}

Records of all patients who had undergone a vitrectomy and silicone oil procedure in Addenbrookes hospital followed by intracapsular cataract surgery with retention of the silicone, the second procedure having been carried out between July 1986 and December 1988 , were examined particularly to identify the occurrence of pupil block with or without raised intraocular pressure, silicone oil in the anterior chamber or silicone oil keratopathy.

The technique of cataract extraction was as follows. Under general anaesthesia a superior Ophthalmology, Clinic 3, Addenbrookes Hospital, Hills Road, Cambridge. 
Table I Indications for silicone oil

\begin{tabular}{lc}
\hline Diagnosis & $\begin{array}{c}\text { Number of } \\
\text { patients }\end{array}$ \\
\hline Proliferative vitreoretinopathy & 21 \\
Proliferative diabetic retinopathy & 10 \\
Giant tear & $7^{*}$ \\
Trauma & 1 \\
Retinal angiomatosis with & 1 \\
$\quad$ proliferative vitreoretinopathy & 1 \\
\hline
\end{tabular}

* includes two patients with Stickler's syndrome

corneal incision was usually followed by two peripheral iridectomies made at 11 o'clock and 1 o'clock using de Wecker's scissors. Very small apertures were avoided. Chymotrypsin was injected through the iridectomies and after two minutes the lens could usually be expressed and only rarely was a cryoprobe or other instrumentation necessary. Loss of silicone oil, if it occurred was recorded. Anterior vitreous remnants or membranes were removed so that in every case the silicone bubble had access to the pupillary aperture. If silicone entered the anterior chamber after extraction it was not removed or replaced in the knowledge that silicone surface tension would allow it to return behind the pupil very soon after surgery.

\section{Results}

Forty patients, 23 males and 17 females, aged between 19 and 86 years (mean 49) had cataract extraction with silicone oil preservation during the study period. $2000 \mathrm{cs}$ silicone oil had been used throughout. The indications for silicone oil are listed in Table I.

Thirty-eight patients had had double superior peripheral iridectomies as described above. One patient had a single superior iridectomy and the case record of one patient was unclear. In one patient the cataract extraction preceded the vitrectomy-silicone oil procedure. Follow up ranged from one to 30 months (median nine months).

In no case was there significant loss of silicone oil.

Two patients developed an acute increase in intra-ocular pressure $(36 \mathrm{mmHg}$ and $45 \mathrm{mmHg}$ ) and had anterior chambers completely filled with silicone oil, this complication occurred at six weeks and eight months after cataract surgery respectively. In each case there were two superior iridectomies which appeared to be occluded by proliferative membrane. Creation of superior and inferior iridotomies with the YAG laser allowed the anterior chamber to refill with aqueous and the pressure to fall in each case. No patient had silicone oil keratopathy.

The results are summarised in Table II.

\section{Discussion}

One serious drawback to the use of liquid silicone in aphakic eyes is that it can cause pupil block and be forced into the anterior chamber resulting in an early post-operative rise in intraocular pressure by open angle trabecular blockage, or a characteristic keratopathy in the long term if constant endothelial contact is maintained. ${ }^{9,10}$

The inferior iridectomy described by Ando and Zivojnovic has resulted in a reduction in frequency of these complications. ${ }^{5}$ They found that keratopathy was almost universal in aphakic patients with silicone oil operated on before this technique (11 of 16 eyes), compared to only six of 62 eyes with an inferior iridectomy. It is not stated whether any patients with an inferior iridectomy developed acute glaucoma due to pupil block by silicone, but four had silicone oil in the anterior chamber in

Table II Complications due to pupil block by silicone oil (no. of patients)

Silicone oil in anterior

chamber with acute rise

of intraocular pressure
Silicone keratopathy
Interval between operations (median)
Intracapsular cataract extraction after vitrectomy and silicone oil procedure (39 patients)

Vitrectomy and silicone oil procedure after cataract extraction (1 patient)
2

0

18 months

(range 1 to 144 )

0
0

2 months 
contact with corneal endothelium at the end of the observation period and another patient with oil in the anterior chamber had YAG laser to re-open the iridectomy so that the oil could move back into the posterior segment. This supports the desirability of an iridectomy. However they speculate that silicone oil, being less dense than water, would tamponade a superior peripheral iridectomy. Laganowski and Leaver also conclude from their retrospective series of 44 patients that a patent inferior peripheral iridectomy is effective in reducing the anterior segment complications of silicone oil in the aphakic eye. ${ }^{11}$

Our results show that using a superior peripheral iridectomy results in a very low incidence of pupil block and that silicone does not readily occlude a superior iridectomy. Moreover, the two patients who developed pupil block demonstrated proliferation over the iridectomy rather than tamponade by silicone. One possible explanation is that silicone oil does not easily enter recesses. This physical characteristic has been demonstrated in rabbit and cat silicone tolerance experiments where silicone could not be made to enter the iridocorneal angle when injected directly into the anterior chamber. ${ }^{12}$ It is improbable that a silicone bubble would conform to the recess of the ciliary sulcus in the manner suggested ${ }^{5}$ and there is more likely to be a meniscus of aqueous between the bubble and the recess.

Also, after cataract extraction performed as a second operation, as in all but one patient in our series and unlike the Rotterdam/ Nagoya patients ${ }^{5}$ who were aphakic at the time of silicone injection, the relative silicone fill is reduced, not because silicone is lost during the extraction, which is rare, but because the volume of the lens is now occupied by aqueous. This may reduce the likelihood of pupil block if the silicone does not completely contact the pupillary aperture, and so a comparison with the Rotterdam/ Nagoya series is not strictly valid. Eight patients in Laganowski and Leaver's series had lens extraction and an inferior peripheral iridectomy subsequent to silicone oil and would be more comparable, but complications were not reported separately for this group. ${ }^{11}$

No prospective comparison between infer- ior and superior peripheral iridectomies has been reported, however it can be estimated from the likely complication rate that such a trial would need to enter at least 200 patients in each group to have a $50 \%$ chance of showing a significant difference were one to exist, even assuming a doubling of the complication rate to be the least difference between the techniques which is clinically important. ${ }^{13^{*}}$

The use of a superior peripheral iridectomy allows the cataract extraction to be performed in the standard intracapsular manner and does not require the use of a suction cutter. Superior iridectomies are also cosmetically more acceptable. Nevertheless, we have not ruled out the need for an inferior peripheral iridectomy in cases where lensectomy is combined with vitrectomy and silicone oil injection.

In conclusion, superior peripheral iridectomy is associated with a low incidence of pupil block when cataract extraction with preservation of silicone oil is performed as a second procedure.

References

${ }^{1}$ Cibis PA, Becker B, Okun E, Canaan S: The use of liquid silicone in retinal detachmentsurgery. Arch Ophthalmol 1962, 68: 590-99.

${ }^{2}$ Scott JD: A rationale for the use of liquid silicone. Trans Ophthalmol Soc UK 1977, 97: 235-7.

${ }^{3}$ Aaberg TM: Management of anterior and posterior proliferative vitreoretinopathy. XLV Edward Jackson Memorial Lecture. Am J Ophthalmol 1988, 106: 519-32.

${ }^{4}$ Ando F: Intraocular hypertension resulting from pupillary block by silicone oil. Am J Ophthalmol 1985, 99: 87-8.

${ }^{5}$ Beekhuis WH, Ando F, Zivojnovic R, Mertens DAE, Peperkamp E: Basal iridectomy at 6 o'clock in the aphakic eye treated with silicone oil. Prevention of keratopathy and secondary glaucoma. Br J Ophthalmol 1987, 71: 197-200.

${ }^{6}$ Zborowsky-Gutman L, Treister G, Naveh N, Chen $\mathrm{V}$, Blumenthal M: Acute glaucoma following vitrectomy and silicone oil injection. Br J Ophthalmol 1987, 71: 903-6.

${ }^{7}$ Sternberg P Jnr, Hatchell DL, Foulks GN, Landers MB III: The effect of silicone oil on the cornea. Arch Ophthalmol 1985, 103: 90-4.

${ }^{8}$ Beekhuis WH, van Rij G, Zivojnovic R: Silicone oil keratopathy: indications for keratopathy. $\mathrm{Br} J$ Ophthalmol 1985, 69: 247-53.

${ }^{9}$ Leaver PK, Grey RHB, Garner A: Silicone oil injection in the treatment of massive preretinal retraction II. Late complications in 93 eyes. $\mathrm{Br} J$ Ophthalmol 1979, 63: 361-7.

${ }^{10}$ Federman JL and Schubert HD: Complications associated with the use of silicone oil in 150 eyes 
after retinavitreous surgery. Ophthalmology 1988, 95: 870-6.

${ }^{11}$ Laganowski HC and Leaver PK: Silicone oil in the aphakic eye: the influence of a six o'clock peripheral iridectomy. Eye 1989, 3: 338-48.
${ }^{12}$ Armaly MF: Ocular tolerance to silicones. Arch Ophthalmol 1962, 68: 390-5.

${ }^{13}$ Miller DK and Homan SM: Graphical aid for determining power of clinical trials involving two groups. Br Med J 1988, 297: 672-6. 